\title{
SOLITONS, DISORDER AND CHARGE CONDUCTION IN NEARLY METALLIC POLYACETYLENE
}

\author{
A. J. EPSTEIN, R. W. BIGELOW, A. FELDBLUM and H. W. GIBSON \\ Xerox Webster Research Center - W114, Webster, NY 14580 (U.S.A.) \\ D. M. HOFFMAN* \\ Department of Physics, Ohio State University, Columbus, OH 43210 (U.S.A.) \\ D. B. TANNER \\ Department of Physics, University of Florida, Gainesville, FL 32611 (U.S.A.)
}

\begin{abstract}
Polyacetylene doped in the range one to five per cent has been shown to have low susceptibility yet high conductivity. A coordinated study of the structural, optical, magnetic and transport properties of polyacetylene doped with iodine to these nearly metallic levels shows that essentially all charges go into soliton-like states. The measurements are in quantitative agreement with charge transport via variable range hopping among soliton-like levels. A parallel conduction mechanism of thermal activation of charge carriers to 'high mobility' extended band states is proposed, and may dominate in less disordered samples.
\end{abstract}

\section{Introduction}

The transformation of polyacetylene, $(\mathrm{CH})_{x}$, from a semiconductor to a metal-like material upon doping with donors or acceptors has been under intense study for the past several years $[1,2]$. The soliton model $[3,4]$ has been very successful in explaining many of the transport, spectroscopic and magnetic properties of polyacetylene, especially in the lightly doped regime [5 - 12]. The nearly metallic region of one to five per cent doping $(0.01 \leqslant$ $y \leqslant 0.05$ ) is of particular interest. Here, the magnetic susceptibility of samples carefully doped with iodine [8], perchlorate [13], arsenic pentafloride [9] or sodium [14] is substantially lower than that of metallic polyacetylene $(y \geq 0.06)$ yet the conductivity $(\sigma)$ is 'nearly metallic' $\left(10^{2} \geq \sigma \geq\right.$ $10^{-1} \mathrm{ohm}^{-1} \mathrm{~cm}^{-1}$ ). This result has led to a variety of suggestions for the

*Present address: Department of Physics, University of Kentucky, Lexington, KY 40506, U.S.A. 
charge conduction mechanism in this regime, including: variable range hopping [15] among soliton-like centers [16], collective transport by mobile spinless charged solitons [14] and interchain hopping of bipolarons [17]. In this paper we demonstrate the important central role of charged solitons using iodine-doped polyacetylene as the model. We show how, under some conditions, disorder will dominate and charge conduction will be via variable range hopping. Finally, we introduce a parallel conduction path to account for variations from sample to sample and dopant to dopant: thermal activation of charge carriers to 'high mobility' extended states.

\section{Experimental methods}

Free standing polyacetylene films were prepared by the Shirakawa technique [18]. The films were $\sim 90 \%$ crystalline [19] and were composed of $\sim 500 \AA$ diameter fibrils [20]. The samples were doped with iodine using the 'slow doping' technique $[8,9]$. Room-temperature reflectance measurements [21] were made between 20 and $20000 \mathrm{~cm}^{-1}$ and Kramers-Kronig analysis gave the frequency $(\omega)$-dependent conductivity, $\sigma(\omega)$. The temperature $(T)$-dependent magnetic susceptibility $(\chi)$ was measured from 4 to 320 $\mathrm{K}$ with a calibrated [8] Faraday technique. The temperature-dependent thermopower $S(T)$ and the four-probe $\sigma(T)$ were measured from 10 to $300 \mathrm{~K}$ with an apparatus that allows these quantities to be determined in the same run.

\section{Optical properties}

The $\sigma(\omega)$ for $y=0.033,0.042$ and 0.048 are shown in Fig. 1 for frequencies up to $6000 \mathrm{~cm}^{-1}$. Extension to $20000 \mathrm{~cm}^{-1}$ for $y=0.042$ shows that neither the interband transition at $\sim 12000 \mathrm{~cm}^{-1}$ nor the strong midgap absorption observed [7] at $\sim 6000 \mathrm{~cm}^{-1}$ is present. Instead, a single intense peak at $3000-3500 \mathrm{~cm}^{-1}$ is found. The far-infrared conductivity obtained is in good agreement with the measured d.c. conductivity. The broad maximum in $\sigma(\omega)$ at $900 \mathrm{~cm}^{-1}$ and the narrow peak at $1370 \mathrm{~cm}^{-1}$ have been associated with charged solitons created by chemical doping [5,6]. The oscillator strength of these features (calculated after subtraction of the lowenergy tail of the $\sim 3000 \mathrm{~cm}^{-1}$ absorption) is plotted versus doping concentration in the inset in Fig. 1. The straight lines are extensions of the strengths of these absorptions at low doping levels [5,22], $y<0.01$. Because the strength per dopant molecule is unchanged up to $y=0.048$, we conclude that doping proceeds through the formation of charged solitons even for doping levels approaching five per cent. This value is twice the critical concentration measured [23] for the divergence of the dielectric constant $\left(y_{c}=\right.$ 0.022 ) in similarly iodine-doped samples. This dielectric divergence may then correspond to the formation of a soliton lattice [24] or Anderson transition [25] at $y_{\mathrm{c}}$. 


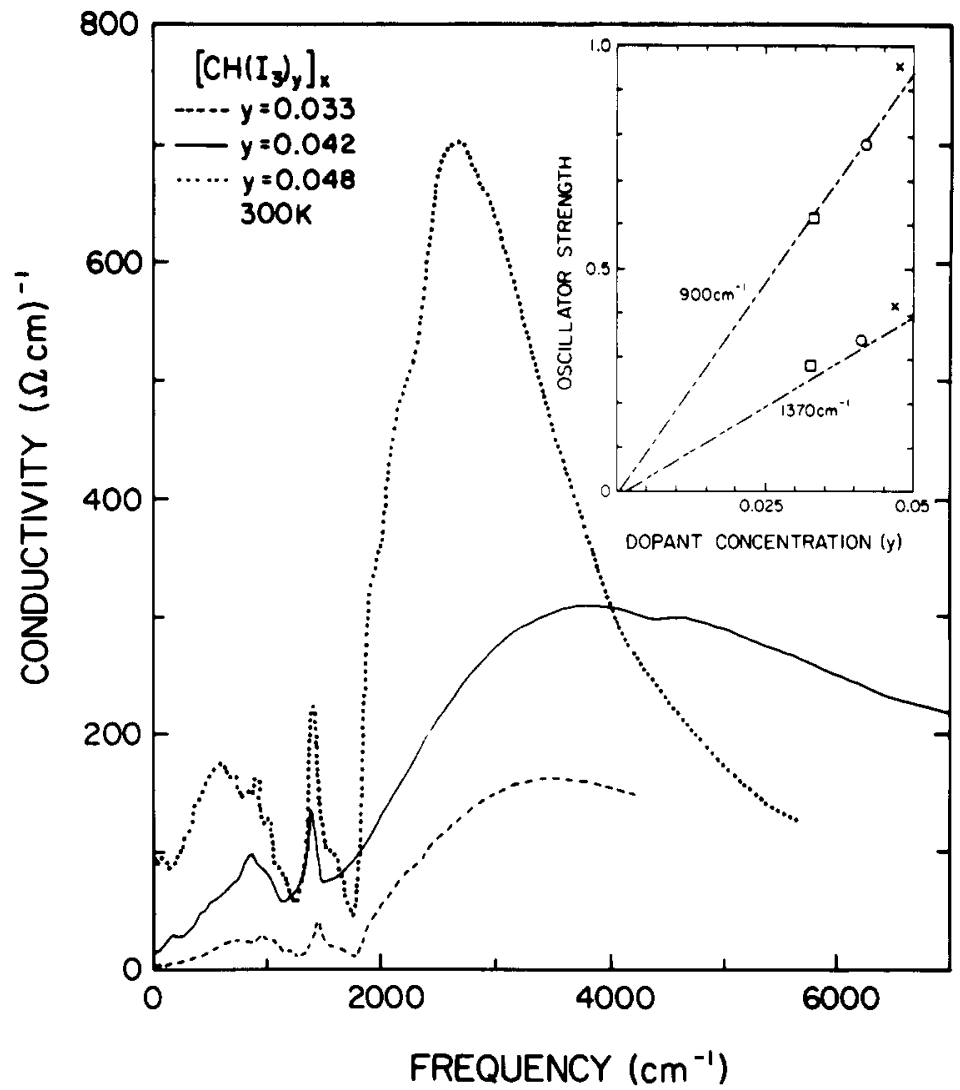

Fig. 1. Conductivity versus frequency for $\left[\mathrm{CH}\left(\mathrm{I}_{3}\right)_{y}\right]_{x}$ (from reflectance data). Inset: the oscillator strength vs. $y$ of the 900 and $1370 \mathrm{~cm}^{-1}$ peaks. The solid lines are extrapolations of the earlier results [22] for $y<0.01$.

The broad mode centered at $900 \mathrm{~cm}^{-1}$ for lightly doped samples has been identified as the 'pinned' mode of the charged soliton bound to a charged impurity $[6,26]$. Examination of Fig. 1 shows that for these iodinedoped samples, the pinned mode remains at $900 \mathrm{~cm}^{-1}$ up to $y=0.042$, but shifts to $\sim 700 \mathrm{~cm}^{-1}$ for $y=0.048$. This supports the idea that the charged solitons remain pinned to the dopant position and do not diffuse independently of the dopant. At low frequencies the room temperature optical conductivity equals the measured d.c. conductivity, in agreement with the prediction of variable range hopping [27].

\section{Magnetic properties}

The $\chi(T)$ is a sum of $\chi^{\text {Curie }}$ and $\chi^{\text {Pauli }}=2 \mu_{\mathrm{B}}^{2} N\left(E_{\mathrm{F}}\right)$, where $\mu_{\mathrm{B}}$ is the Bohr magneton and $N\left(E_{F}\right)$ the density of states at the Fermi level. Note that $(\mathrm{CH})_{x}$ with zero band gap would have $[8,9,28] N\left(E_{\mathrm{F}}\right) \sim 0.16-0.20$ states/eV C 
TABIE 1

Summary of results for $\left[\mathrm{CH}\left(\mathrm{I}_{3}\right)_{y}\right]_{x}$

\begin{tabular}{lllllll}
\hline $\begin{array}{l}\text { Starting } \\
(\mathrm{CH})_{x}\end{array}$ & $y$ & $\begin{array}{l}N\left(E_{\mathrm{F}}\right) \\
(\text { states/eV C) }\end{array}$ & $\begin{array}{l}\sigma(295 \mathrm{~K}) \\
\left(\Omega^{-1} \mathrm{~cm}^{-1}\right)\end{array}$ & $\begin{array}{l}T_{0} \\
\left(10^{5} \mathrm{~K}\right)\end{array}$ & $\begin{array}{l}\alpha^{-1} \\
(\AA)\end{array}$ & $\begin{array}{l}\sigma_{\mathrm{H}} \\
\left(\Omega^{-1} \mathrm{~cm}^{-1}\right)\end{array}$ \\
\hline trans & 0.017 & 0.014 & 2.5 & 19 & 5.2 & 1.9 \\
cis & 0.033 & 0.045 & 8 & 29 & 3.1 & 9.1 \\
trans & 0.042 & 0.027 & 20 & 3.8 & 7.1 & 3.1 \\
cis & 0.048 & 0.069 & 50 & 4.6 & 4.9 & 12 \\
\hline
\end{tabular}

atom, while Table 1 shows that $N\left(E_{\mathrm{F}}\right)$ for the two initially trans-doped samples is much smaller, nearly as low as that reported earlier $[8,9]$ for lowsusceptibility, slowly doped samples. Though small, the measured $N\left(E_{\mathrm{F}}\right)$ is much larger than that of an uncompensated amorphous semiconductor [29] such as amorphous $\mathrm{Si}$. The finite $N\left(E_{\mathrm{F}}\right)$ is in accord with the phase disordering of the $(\mathrm{CH})_{x}$ Peierls insulator due to the aperiodic location of the dopants, where $N\left(E_{\mathrm{F}}\right)$ is non-zero due to states being pulled out of the valence and soliton bands by the $\mathrm{I}_{3}$ disorder [28]. These states should retain soliton-like character. Figure 2 (a) is a schematic diagram of the resulting electronic structure of the iodine-doped nearly metallic polyacetylene.

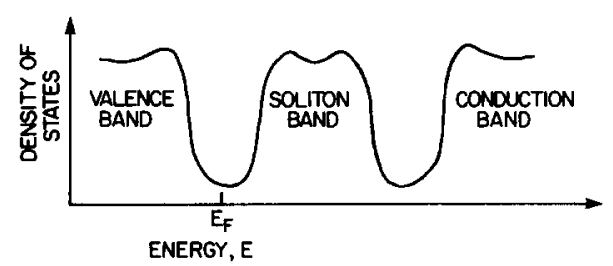

(a)

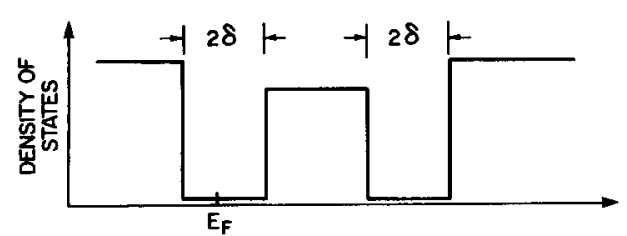

(b)

ENERGY, E

Fig. 2. Schematic electronic structure for doped polyacetylene. The Fermi energy is shown for acceptor doping. (a) Disorder strong; (b) disorder weak. $2 \delta$ is the energy gap between the valence (conduction) band and the soliton band.

\section{Structural disorder}

X-ray diffraction measurements [30] of cis- $(\mathrm{CH})_{x}$ slowly doped to $y=$ 0.033 and $y=0.048$ show the same pattern as those for trans-(CH) $)_{x}$ slowly 
doped to $y=0.017$ and $y=0.042$. This result is in agreement with earlier optical [22] and transport [31] studies which indicated that the doping of cis $-(\mathrm{CH})_{x}$ resulted in doped trans $-(\mathrm{CH})_{x}$. For all iodine doping levels studied [30], about one-third of the sample volume remains undoped, probably in the centers of the fibrils. Three broad X-ray reflections are found for $\left[\mathrm{CH}\left(\mathrm{I}_{3}\right)\right]_{x}$ for interplanar spacings of $7.9 \AA, 4.1 \AA$ and $2.9 \AA$, in agreement with a model [31] of intercalation between polyacetylene planes. The transverse coherence length for the doped $(\mathrm{CH})_{x}$ is $20 \pm 5 \AA$.

The coherence observed in the X-ray structural studies suggests that a side view of the iodine-doped $(\mathrm{CH})_{x}$ system is as schematically shown in Fig. 3. The actual extent of the disorder, and hence the magnitude of $N\left(E_{\mathrm{F}}\right)$, is expected to be a sensitive function of initial polyacetylene state (cis-trans content, crystallinity, fibril diameter, crosslinking, etc.), doping conditions (vapor pressure, soliton concentration or electrochemical potential, temperature, etc.) and dopant (dopant size, shape, reactivity, etc.). For example, $\chi\left[N\left(E_{\mathrm{F}}\right)\right]$ of the doped initially cis-(CH) films, while smaller than the zero bandgap value, is generally larger than that of trans- $(\mathrm{CH})_{x}$ doped to approximately the same concentration $[16,33]$. This may be due to increased disorder in the doped cis because of the large structural charges that accompany doping-induced isomerization, leading to a higher $N\left(E_{\mathrm{F}}\right)$. In comparison, doping in trans is an intercalation process [32] that may introduce less disorder and hence lower $N\left(E_{F}\right)$ [34].

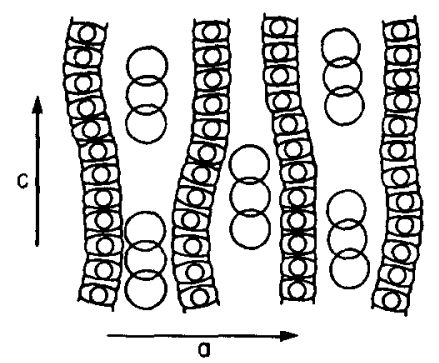

Fig. 3. Schematic view of the ac plane of $\left[\mathrm{CH}\left(\mathrm{I}_{3}\right)_{y}\right]_{x}$. The X-ray data show only a $20 \pm$ $5 \AA$ coherence length perpendicular to the chain axis [30].

\section{Transport properties}

Because $N\left(E_{\mathrm{F}}\right)$ is below the metallic value yet very high for a semiconductor, we have analyzed $\sigma(T)$ within the variable-range hopping (VRH) model [29]. This model gives

$\sigma(T)=\left(0.39\left[N\left(E_{\mathrm{F}}\right) / \alpha k_{\mathrm{B}} T\right]^{1 / 2} \nu_{0} e^{2}\right) \exp \left[-\left(T_{0} / T\right)^{1 / 4}\right]$,

where $T_{0}=16 \alpha^{3} / k_{\mathrm{B}} N\left(E_{\mathrm{F}}\right), \alpha^{-1}$ is the decay length of a localized state, and $\nu_{0}$ is a hopping attempt frequency. The fit of $\sigma(T)$ by the functional form $T^{-1 / 2} \exp \left[-\left(T_{0} / T\right)^{1 / 4}\right]$ is shown as a solid line for each sample in Fig. 4, with 


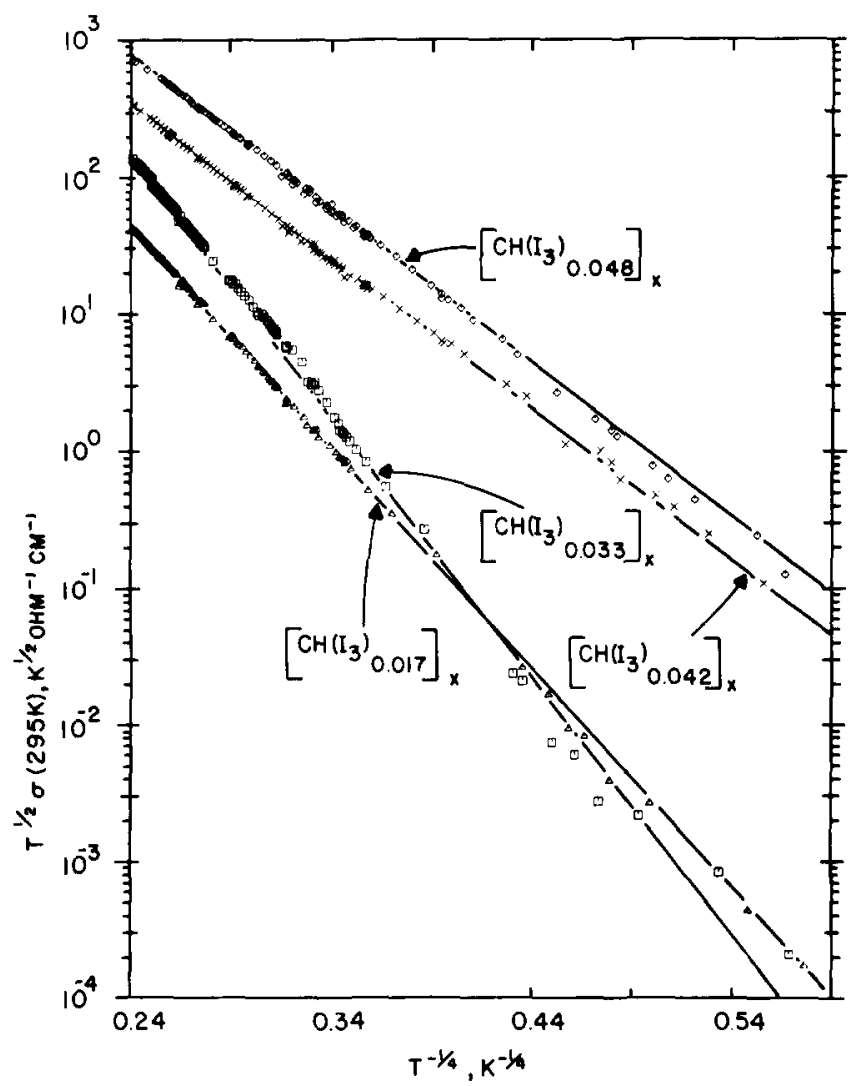

Fig. 4. $T^{1 / 2} \sigma(T) / \sigma(295 \mathrm{~K})$ us. $T^{-1 / 4}$ slow-doped $\left[\mathrm{CH}\left(\mathrm{I}_{3}\right)_{y}\right]_{x}$. The solid lines are from theoretical fits described in the text (eqn. (1)).

the one adjustable parameter, $T_{0}$, given in Table 1 . Fits with other behaviors, e.g., $\exp \left[-\left(T_{0} / T\right)^{m}\right]$ with $m$ other than $1 / 4$, were not as good. Using the measured values of $N\left(E_{\mathrm{F}}\right)$ from $\chi$ and $T_{0}$ from $\sigma$, we calculate $\alpha^{-1} \approx 5 \AA$, varying slightly from sample to sample (see Table 1 ). Then, assuming that [35] $v_{0}=\nu_{\mathrm{ph}} \exp (2 \alpha R)$, where $R$ is the separation between hopping sites and $\nu_{\mathrm{ph}}$ is an optical phonon frequency $\left(\sim 3.6 \times 10^{13} \mathrm{~s}^{-1}\right)$, the conductivities calculated from eqn. (1) ( $\sigma_{\mathrm{H}}$ in Table 1$)$ are in good agreement with experiment. The decay length $\alpha^{-1}$ evaluated for these samples is consistent with the effective dimensionally averaged decay length of a non-interacting soliton $[10,36], \xi=\left(\xi_{\lrcorner}{ }^{2} \xi_{\|}\right)^{1 / 3}=3.6 \AA$. This picture is also consistent with $N\left(E_{\mathrm{F}}\right)$ being non-zero due to states being pulled out of the valence and soliton bands by the $I_{3}$ disorder [28]. These states should retain a large soliton-like character.

The temperature-dependent thermopower is an independent probe of the specific charge transport mechanism. For example, for VRH [29]

$$
S^{\mathrm{VRH}}(T)=1 / 2\left(k_{\mathrm{B}} / e\right) k_{\mathrm{B}}\left(T_{0} T\right)^{1 / 2}\left(N^{-1} \mathrm{~d} N / \mathrm{d} \epsilon\right)
$$


while for a metal [29],

$S^{\mathrm{m}}(T)=\left(2 \pi^{2} / 3\right)\left(k_{\mathrm{B}} / e\right) k_{\mathrm{B}} T\left(N^{-1} \mathrm{~d} N / \mathrm{d} \epsilon\right)$.

The $S(T)$ was measured on the same samples during the $\sigma(T)$ study $[16,37]$. The best fit [16,37] for $y<0.04$ was with eqn. (2) with $N^{-1} \mathrm{~d} N / \mathrm{d} \epsilon \sim 0.4$ $\mathrm{eV}^{-1}$, in agreement with variable range hopping. For the most highly doped samples, $S(T)$ becomes more nearly linear in $T$, reflecting the nearly delocalized behavior consistent with the larger $\alpha^{-1}$.

\section{Discussion}

Although the disordered Peierls insulator with variable range hopping among soliton-like states is appropriate for these samples doped with $\mathrm{I}_{3}{ }^{-}$in the range $0.01 \leqslant y \leqslant 0.05$, other samples of iodine-doped $(\mathrm{CH})_{x}[38], \mathrm{ClO}_{4}^{-}$doped $(\mathrm{CH})_{x}$ [13] and Na-doped $(\mathrm{CH})_{x}[14]$ in the same concentration range have Pauli susceptibilities (and hence $N\left(E_{\mathrm{F}}\right)$ ) too small for the straightforward application of the VRH model. This result has led to the suggestion of the importance of collective transport by mobile spinless charged solitons [14] and interchain hopping of bipolarons [17].

We present here an alternative approach to understanding the charge conduction in these small $N\left(E_{\mathrm{F}}\right)$ samples: thermal activation of charge carriers to 'high mobility' extended states [39]. As discussed above and illustrated in Fig. 2(a), the phase disordering of the Peierls insulator by the random dopant potentials and reduced crystallinity lead to a finite density of states in the gaps between the valence and conduction bands and the soliton band. The edges of the valence, soliton (condensate) and conduction bands are no longer sharp [40], the states in the band edges and band tails will be localized and the location of the mobility edge will depend on the individual dopant configuration [41]. Because the samples with small $N\left(E_{\mathrm{F}}\right)$ are usually doped in a particularly gentle manner (e.g., slow intercalation in trans- $(\mathrm{CH})_{x}$ and use of very thin $50 \AA$ fibrils), it is plausible that the resulting doped polymer is much less disordered. The corresponding electronic structure is schematically as shown in Fig. 2(b), with the states at the band edges being more 'extended' than those in Fig. 2(a).

For acceptor (donor) doping, thermodynamic equilibrium requires excitation of a finite number of electrons from the occupied valence (soliton) band to the unoccupied soliton (conduction) band, which is located at an energy $2 \delta$ higher. An estimate of the number of charge carriers available at room temperature can be made using reasonable parameters for $N(E)$ and $2 \delta$. For simplicity, we assume here that the density of states for the valence, soliton (or condensate) and conduction bands is independent of $E$ and has the same value in each band. More detailed assumptions for $N(E)$ do not substantially change our results. Assuming [8] the density of states at the band edge is $\sim 0.2$ state/eV C (probably an underestimate because of the one-dimensional band structure) and, from the infrared data (e.g., Fig. 1) 
and magnetic data [8], that $2 \delta=0.3 \mathrm{eV}$, the number of holes (electrons) excited at room temperature is given by

$n(T) \approx N(E) k_{\mathrm{B}} T \exp \left(-\delta / k_{\mathrm{B}} T\right)$

or $n(300 \mathrm{~K})=3 \times 10^{17} \mathrm{~cm}^{-3}$. An equal number of electrons (holes) are excited into the soliton band. A more detailed treatment of carrier excitation in thermal equilibrium is given by Conwell [42]. At this concentration of electrons or holes, the magnetic susceptibility would be below that reported [14] for small $N\left(E_{F}\right)$ samples. For an electrical conductivity [14] of $1 \mathrm{ohm}^{-1} \mathrm{~cm}^{-1}$, and assuming that the holes (electrons) in the valence (conduction) band and the electrons (holes) in the soliton band are equally mobile, $\sigma=n e \mu$ requires a mobility of $11 \mathrm{~cm}^{2} / \mathrm{V} \mathrm{s}$ for the excited charge carriers. This number is quite reasonable; it is even smaller than what is expected for polyacetylene. For comparison, the segregated stack charge transfer salt (tetrathiafulvalenium)-(tetracyanoquinodimethanide) [(TTF) (TCNQ)] has a mobility at $300 \mathrm{~K}$ of $4 \mathrm{~cm}^{2} / \mathrm{V} \mathrm{s}$ [43], while the $300 \mathrm{~K}$ mobility for electrons at the bottom of the conduction band of polyparaphenylene has been calculated [44] to be $\sim 600 \mathrm{~cm}^{2} / \mathrm{V} \mathrm{s}$. The mobility for ordered polyacetylene was expected [44] to be similar to that calculated for polyparaphenylene.

The total measured conductivity of a particular $(\mathrm{CH})_{x}$ sample is then a sum of contributions from hopping at the Fermi level, $\sigma_{\mathrm{H}}$, and activation of carriers to the band edges, $\sigma_{\mathrm{A}}$ :

$\sigma=\sigma_{\mathrm{A}}+\sigma_{\mathrm{H}}$

With increasing disorder, $\sigma_{\mathrm{H}}$ is expected to increase (through increased $N\left(E_{\mathrm{F}}\right)$ ) while $\sigma_{\mathrm{A}}$ decreases (through decreasing $\mu$ ). As a result the conductivity of samples doped under a variety of conditions can remain in the range $10^{-1}$ to $10^{+2} \mathrm{ohm}^{-1} \mathrm{~cm}^{-1}$ for $0.01 \lessgtr y \leqslant 0.05$ despite a widely varying $N\left(E_{\mathrm{F}}\right)$.

\section{Summary}

In summary, the charges in nearly metallic polyacetylene are accommodated in soliton-like states. The detailed coordinated measurements of the structural, optical, magnetic and transport properties of polyacetylene doped with $\mathrm{I}_{3}$ to $0.01 \leqslant y \leqslant 0.05$ are in quantitative agreement with charge transport via variable range hopping among soliton-like levels. It is shown how the conductivity of samples of much lower susceptibility may be accounted for by the thermal activation of carriers to extended states.

\section{Acknowledgements}

This work is funded in part by National Science Foundation Solid State Chemistry Grant No. DMR-8218021. We acknowledge useful discussions with E. M. Conwell, P. Robin, J. P. Pouget and R. Comes. 


\section{References}

1 C. K. Chiang, C. R. Fincher, Jr., Y. W. Park, A. J. Heeger, H. Shirakawa, E. J. Louis, S. C. Gau and A. G. MacCiarmid, Phys. Rev. Lett., 39 (1977) 1098.

2 A. J. Epstein and E. M. Conwell (eds.), Proc. Int. Conf. on Low-Dimensional Conductors, Boulder, CO, Aug. 9-14, 1981, (Mol. Cryst. Liq. Cryst., 77 (1981) and 83 (1982)); see also these Proceedings.

3 M. J. Rice, Phys. Lett., 71 A (1979) 152.

4 W. P. Su, J. R. Schrieffer and A. J. Heeger, Phys. Rev. Lett., 42 (1979) 1698 and Phys. Rev. B, 22 (1980) 2099.

5 C. R. Fincher, Jr., M. Ozaki, A. J. Heeger and A. G. MacDiarmid, Phys. Rev. B, 19 (1979) 4140; E. J. Mele and M. J. Rice, Phys. Rev. Lett., 45 (1980) 926.

6 B. Horovitz, Solid State Commun., 41 (1983) 593, 729.

7 A. Feldblum, A. J. Heeger, T. C. Chung and A. G. MacDiarmid, J. Chem. Phys., 77 (1982) 5114.

8 A. J. Epstein, H. Rommelmann, M. A. Druy, A. J. Heeger and A. G. MacDiarmid, Solid State Commun., 38 (1981) 683.

9 S. Ikehata, J. Kaufer, T. Woerner, A. Pron, M. A. Druy, A. Sivak, A. J. Heeger and A. G. MacDiarmid, Phys. Rev. Lett., 45 (1980) 423.

10 A. J. Epstein, H. Rommelmann, M. Abkowitz and H. W. Gibson, Phys. Rev. Lett., 47 (1981) 1549, and Mol. Cryst. Liq. Cryst., 77 (1981) 81.

11 N. Suzuki, M. Ozaki, S. Etemad, A. J. Heeger and A. G. MacDiarmid, Phys. Rev. Lett., 45 (1980) 1209.

12 D. Moses, A. Denenstein, J. Chen, A. J. Heeger, P. McAndrew, T. Woerner, A. G. MacDiarmid and Y. W. Park, Phys. Rev. B, 25 (1982) 7652.

13 A. Feldblum, J. W. Kaufer, H. W. Gibson and A. J. Epstein, to be published.

14 T. C. Chung, F. Moraes, J. D. Flood and A. J. Heeger, to be published.

15 A. J. Epstein, H. W. Gibson, P. M. Chaikin, W. G. Clark and G. Gruner, Phys. Rev. Lett., 45 (1980) 1730, and Chem. Scr., 17 (1981) 135.

16 A. J. Epstein, H. Rommelmann, R. Bigelow, H. W. Gibson, D. M. Hoffman and D. B. Tanner, Phys. Rev. Lett., 50 (1983) 1866.

17 R. R. Chance, J. L. Brédas and R. Silbey, to be published.

18 T. Ito, H. Shirakawa and S. Ikeda, J. Polym. Sci., Polym. Chem. Ed., 12 (1974) 11; $13(1975) 1942$.

19 P. Robin, J. P. Pouget, R. Comes, H. W. Gibson and A. J. Epstein, Phys. Rev. B, 27 (1983) 3938.

20 A. J. Epstein, H. Rommelmann, R. Fernquist, H. W. Gibson, M. A. Druy and T. Woerner, Polymer, 23 (1982) 1211.

21 K. D. Cummings, D. B. Tanner and J. S. Miller, Phys. Rev. B, 24 (1981) 4142.

22 D. M. Hoffman, H. W. Gibson, A. J. Epstein and D. B. Tanner, Phys. Rev. B, 27 (1983) 1454 .

23 D. M. Hoffman, D. B. Tanner, A. J. Epstein and H. W. Gibson, Mol. Cryst. Liq. Cryst., 83 (1982) 1175.

24 M. Nakahara and K. Maki, Phys. Rev. B, 24 (1981) 1045.

25 S. Kivelson, unpublished results.

26 G. B. Blanchet, C. R. Fincher, T. C. Chung and A. J. Heeger, Phys, Rev. Lett., 50 (1983) 1938.

27 S. Kivelson and A. J. Epstein, Phys. Rev. B, in press.

28 E. J. Mele and M. J. Rice, Phys. Rev. B, 23 (1981) 5397; M. J. Rice and E. J. Mele, Chem. Scr., 17 (1981) 121.

29 N. F. Mott and E. A. Davis, Electronic Processes in Non-Crystalline Materials, Clarendon Press, Oxford, 1979.

30 P. Robin, J. P. Pouget, R. Comes, H. W. Gibson and A. J. Epstein, Polymer, 24 (1983) 1558; J. Phys. (Paris) Colloq., 44 (1983) C3-87.

31 A. J. Epstein, H. Rommelmann and H. W. Gibson, to be published. 
32 S. L. Hsu, A. J. Signorello, G. P. Pez and R. H. Baughman, J. Chem. Phys., 69 (1978) 106.

33 Y. Tomkiewicz, T. D. Schultz, H. B. Brom, A. R. Taranko, T. C. Clarke and G. B. Street, Phys. Rev. B, 24 (1981) 4348.

34 A. J. Epstein, H. Rommelmann, R. W. Bigelow, H. W. Gibson, D. M. Hoffman and D.

B. Tanner, Phys. Rev. Lett., 51 (1983) 2020.

35 R. Colson and P. Nagel, J. Non-Cryst. Solids, 35 (1980) 129.

36 S. Kivelson, Phys. Rev. B, 25 (1982) 3798.

37 A. J. Epstein, H. Rommelmann, R. Bigelow, H. W. Gibson, D. M. Hoffman and D. B. Tanner, J. Phys. (Paris) Colloq., 44 (1983) C3-61.

38 A. Feldblum, R. Bigelow, H. W. Gibson, A. J. Epstein and D. B. Tanner, Mol. Cryst. Liq. Cryst., in press.

39 A. J. Epstein, A. Feldblum, R. W. Bigelow and H. W. Gibson, to be published.

40 D. Vanderbuilt and E. J. Mele, Phys. Rev. B, 22 (1980) 3939.

41 W. P. Su, to be published.

42 E. M. Conwell, to be published.

43 A. J. Epstein, E. M. Conwell and J. S. Miller, Annal. N.Y. Acad. Sci., 313 (1978) 183.

44 C. Menendez and F. Guinea, Phys. Rev. B, 28 (1983) 2183. 